\title{
Photochromic Polyoxometalate/Perylenediimide D-A Hybrid Crystals with Interesting Luminescent Properties
}

Ming-Hua You ${ }^{*}{ }^{*}$, Meng-Hua Li ${ }^{*}$, Yi-Ming Di*, Shu-Quan Zhang ${ }^{\xi}$, and Mei-Jin Lin **\#, *

$†$ College of Materials Science and Engineering, Fujian University of Technology, China, 350118.

$\$$ Key Laboratory of Molecule Synthesis and Function Discovery (Fujian Province University), College of Chemistry, Fuzhou University, Fuzhou, 350108, China. E-mail: meijin_lin@fzu.edu.cn.

§ College of Zhicheng, Fuzhou University, China, 350002.

\# College of Materials Science and Engineering, Fuzhou University, China, 350116

\section{Table of Contents:}

1. Crystallographic data

2. Infrared spectra

3. X-ray powder diffraction analyses

4. Photochromic reversibility

5. Electron spin resonance spectra

6. Transient emission spectra 


\section{Crystallographic data}

Table S1: Crystal data and structure refinement for 1-4

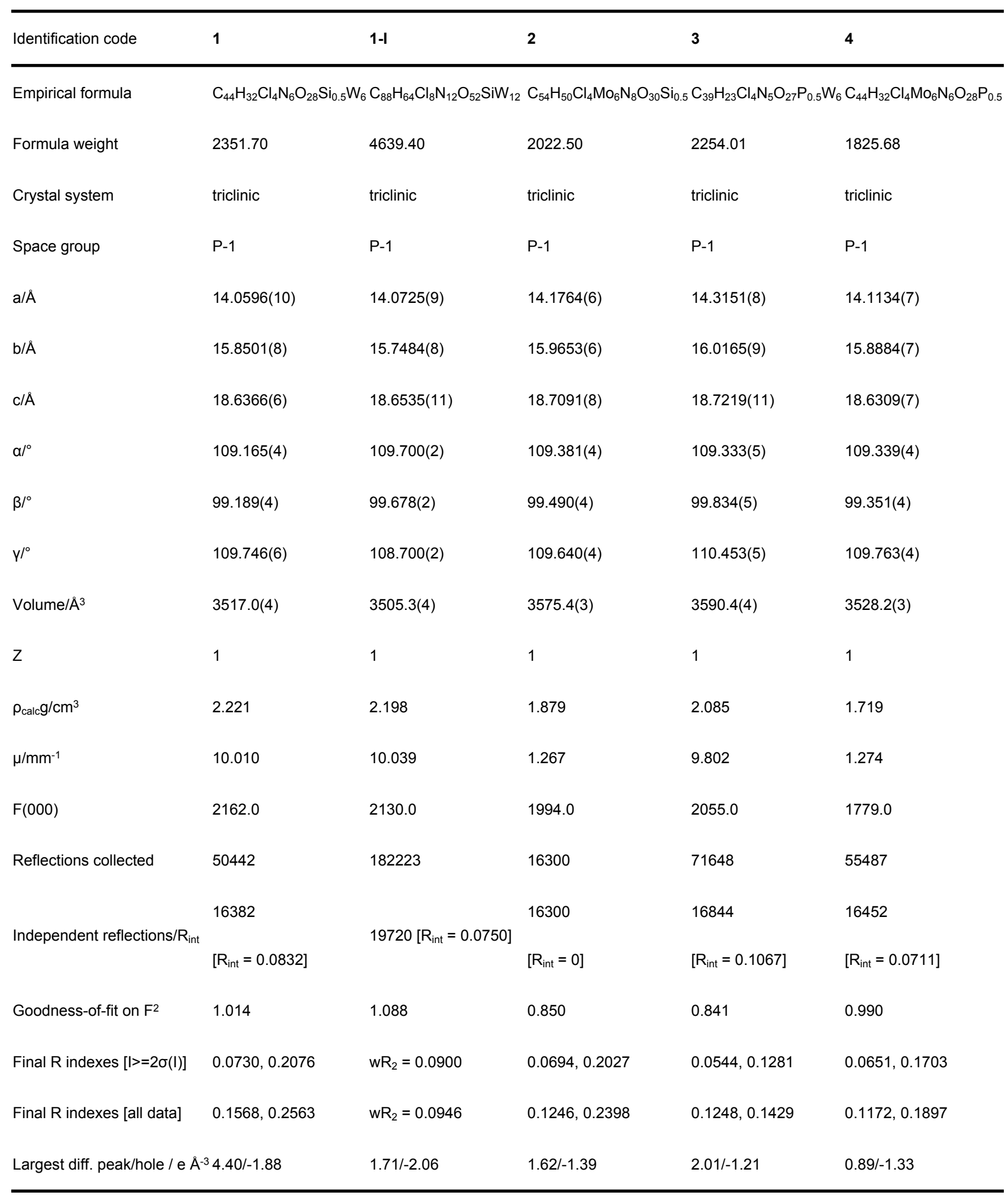




\section{Infrared spectral}
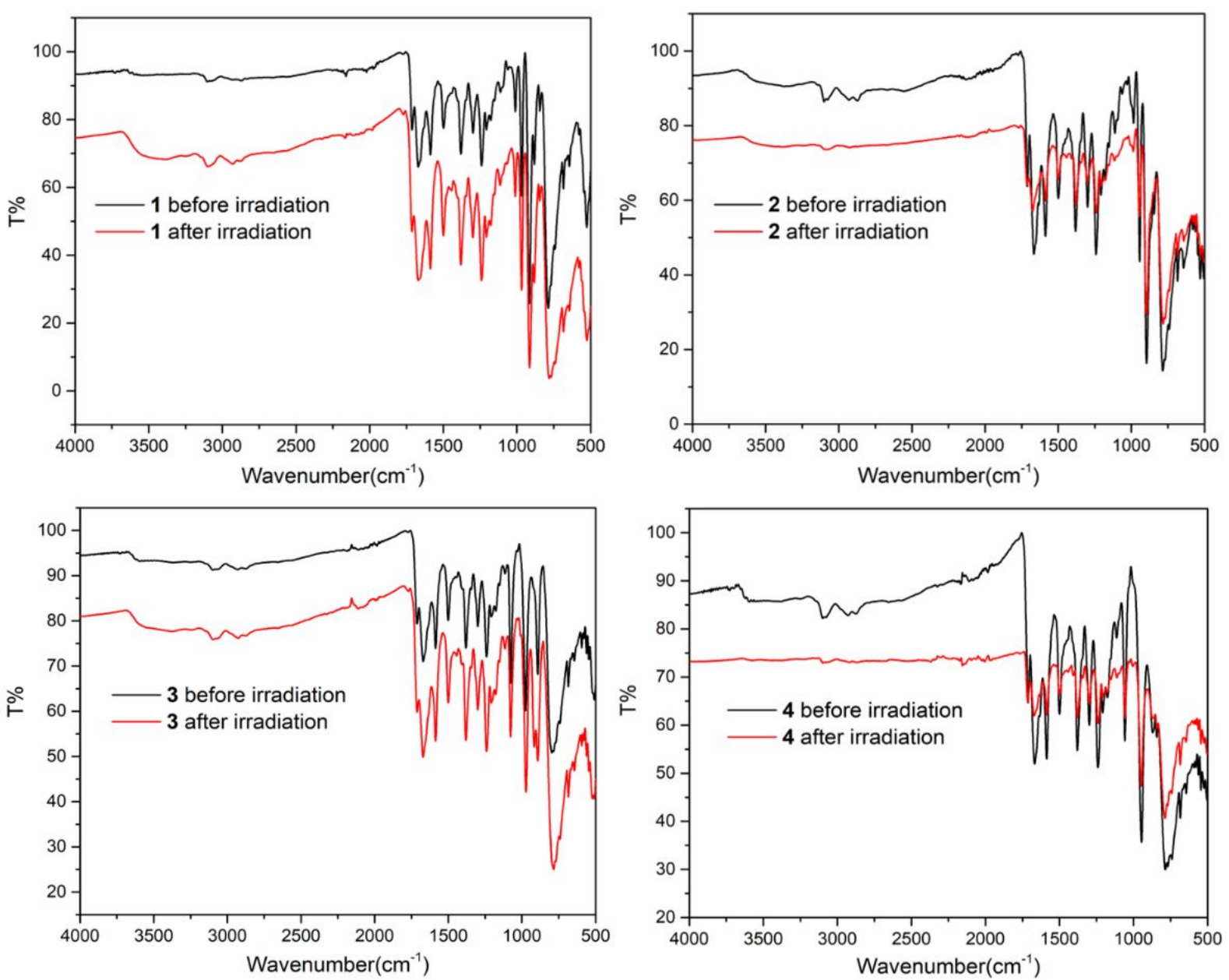

Fig. S1: Infrared spectra of hybrids 1-4 


\section{X-ray powder diffraction analyses}

(a)

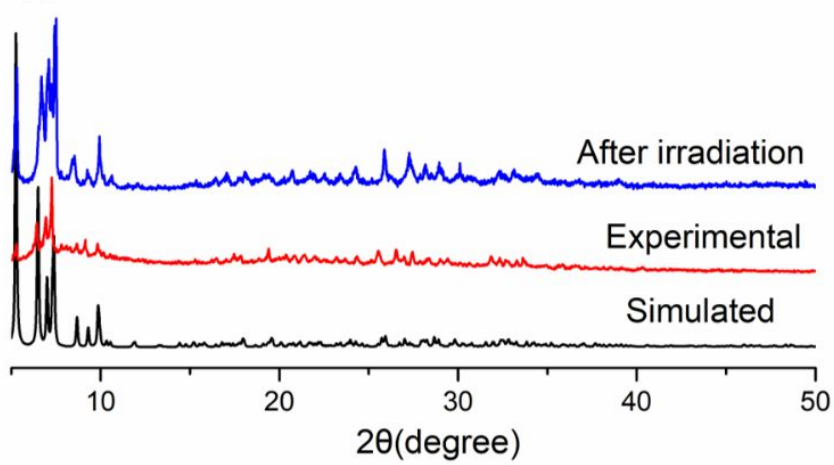

(c)

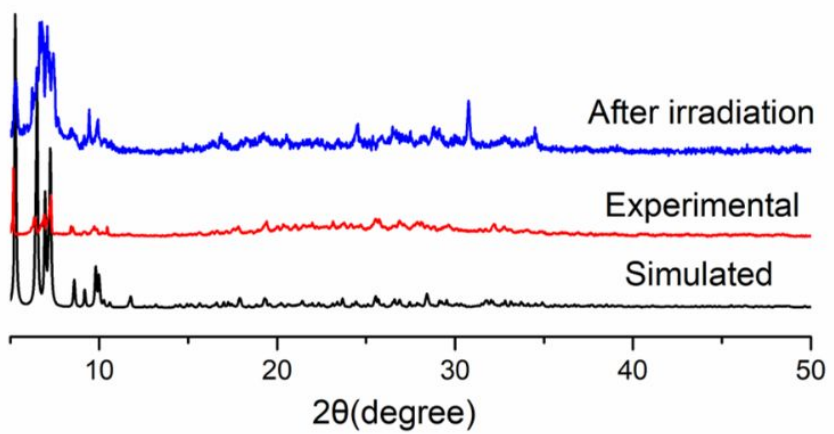

(b)

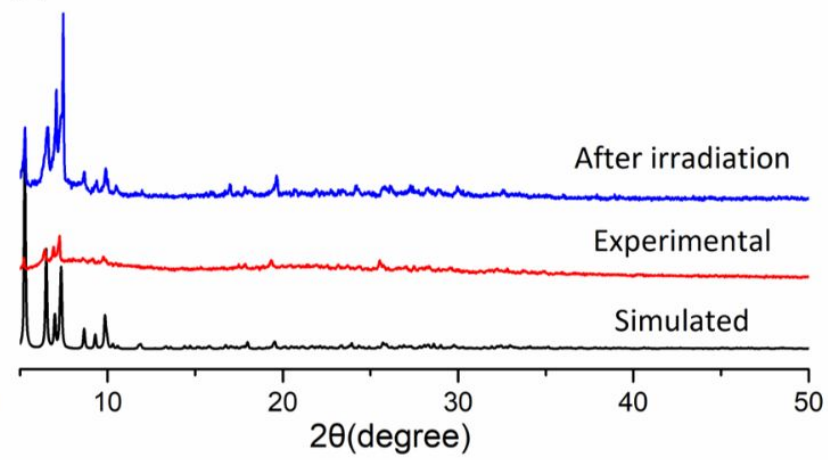

(d)

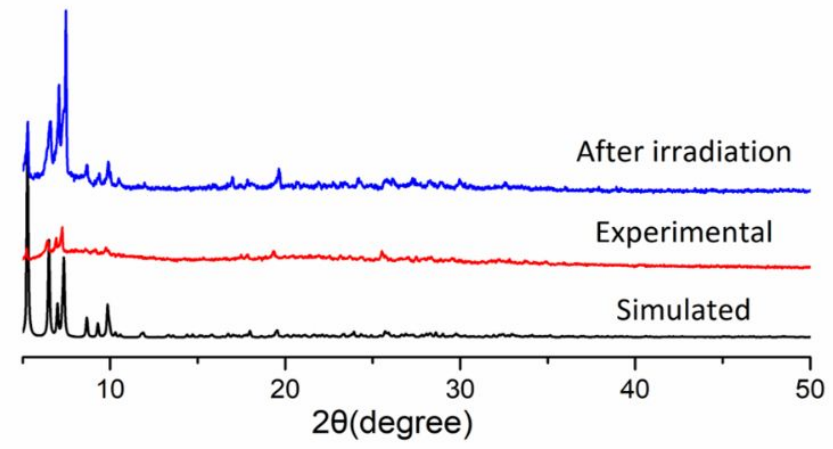

Fig. S2: The observed PXRD patterns for hybrids 1-4 


\section{Photochromic reversibility}

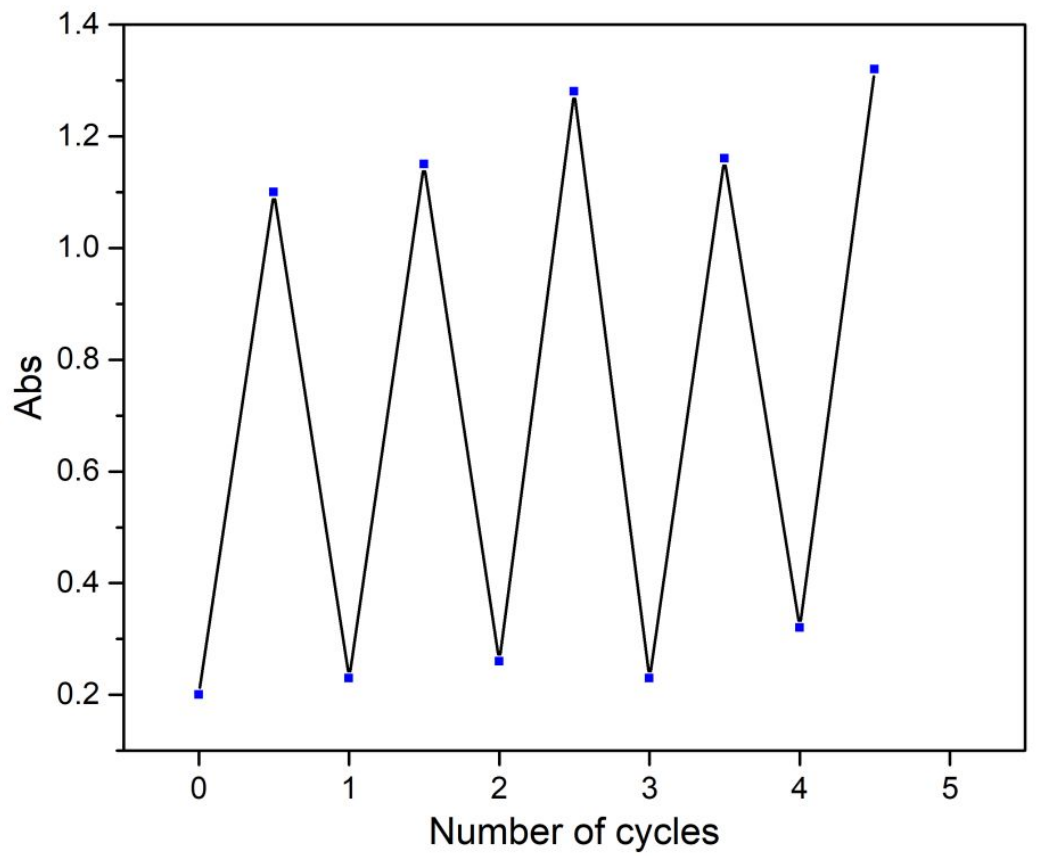

Fig. S3: The UV-vis diffuse-reflectance spectral intensities of hybrid 1 at $780 \mathrm{~nm}$ upon Xenon lamp UV irradiation and under the dark environment alternately 


\section{Electron spin resonance spectra}
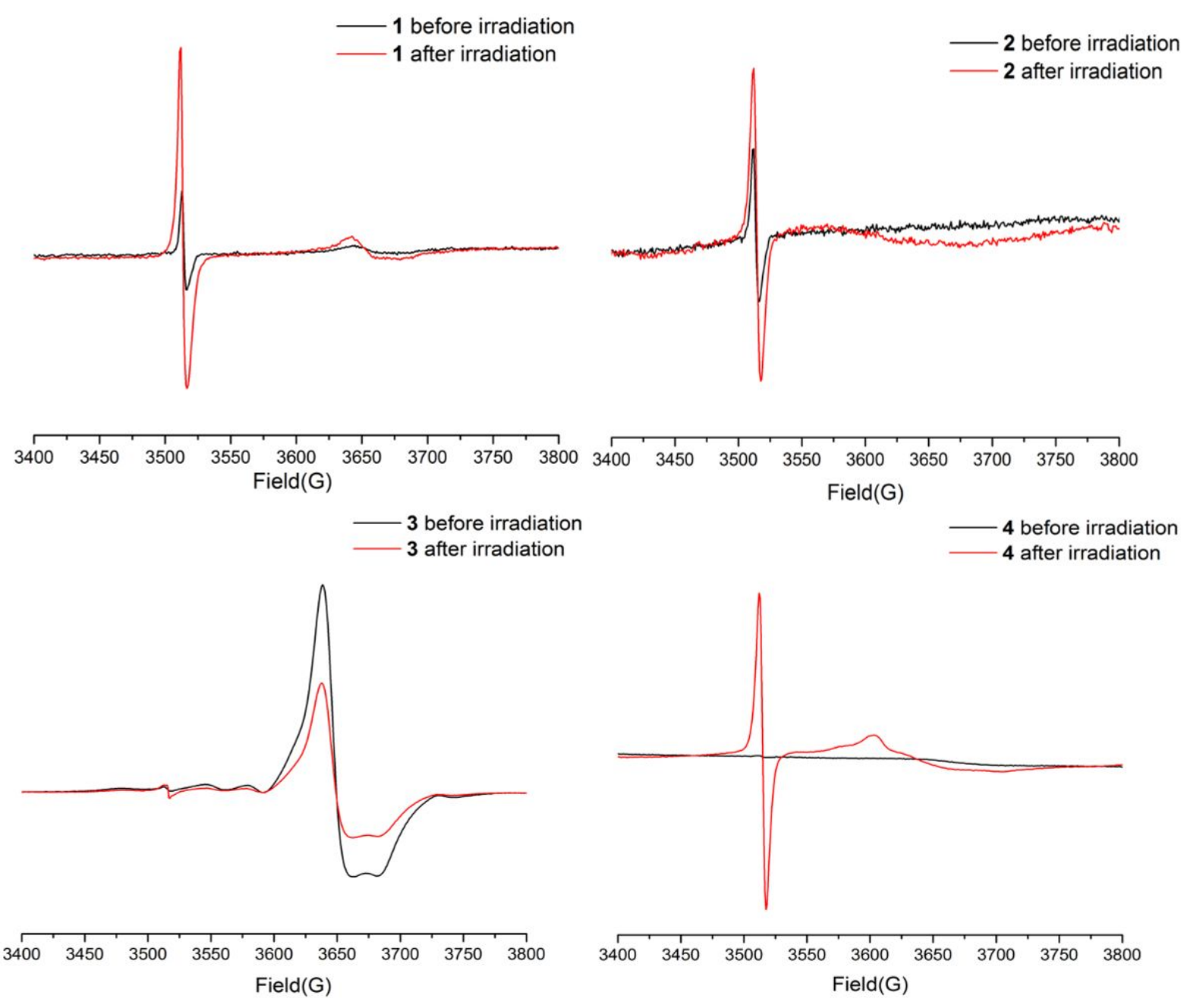

Fig. S4: ESR spectra of hybrids 1-4 before and after irradiation 


\section{Transient fluorescence spectra}
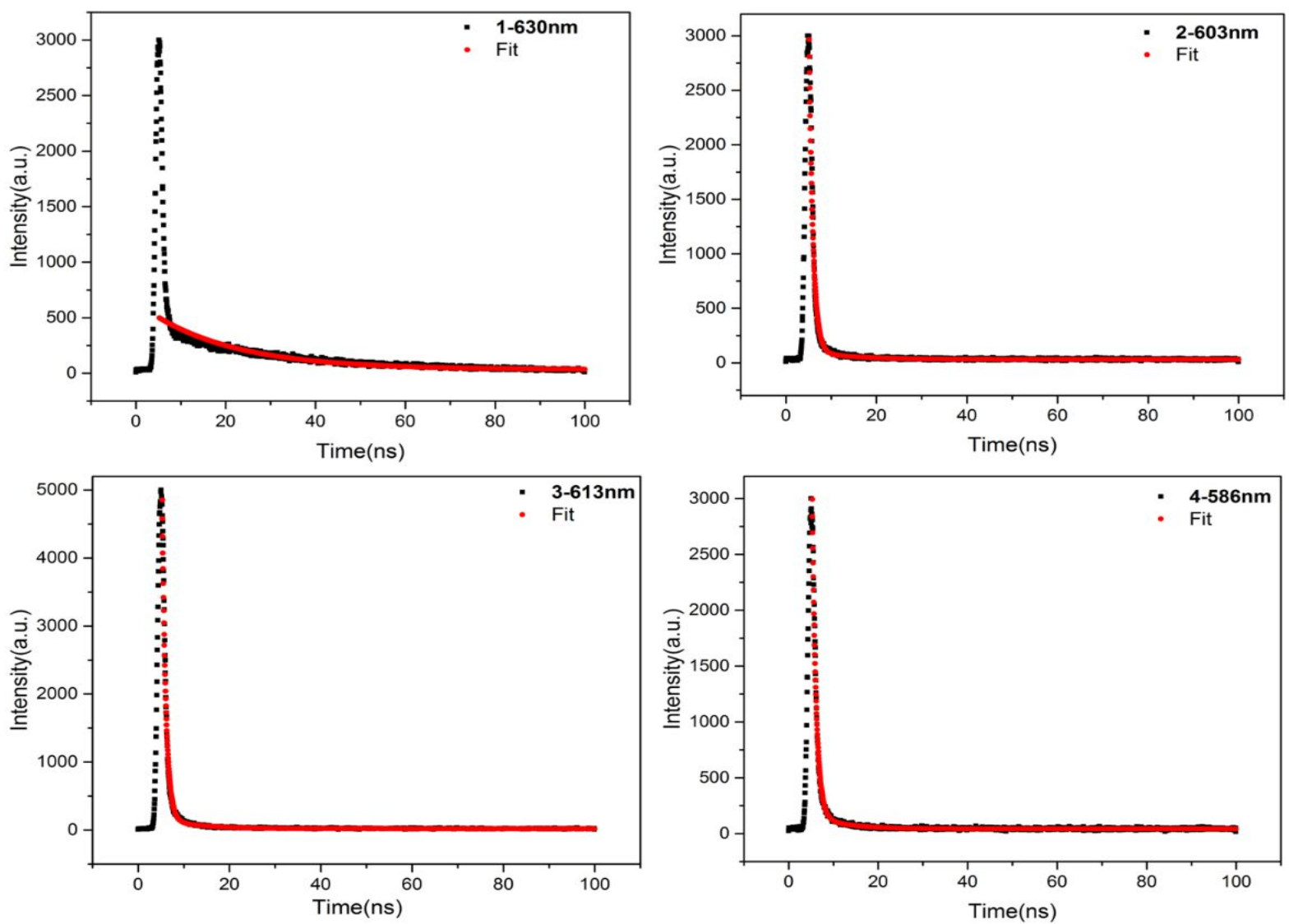

Fig. S5: Luminescence decays of the emissions at the long wavelengths for hybrids 1-4 by using nanosecond lamp under ambient conditions 

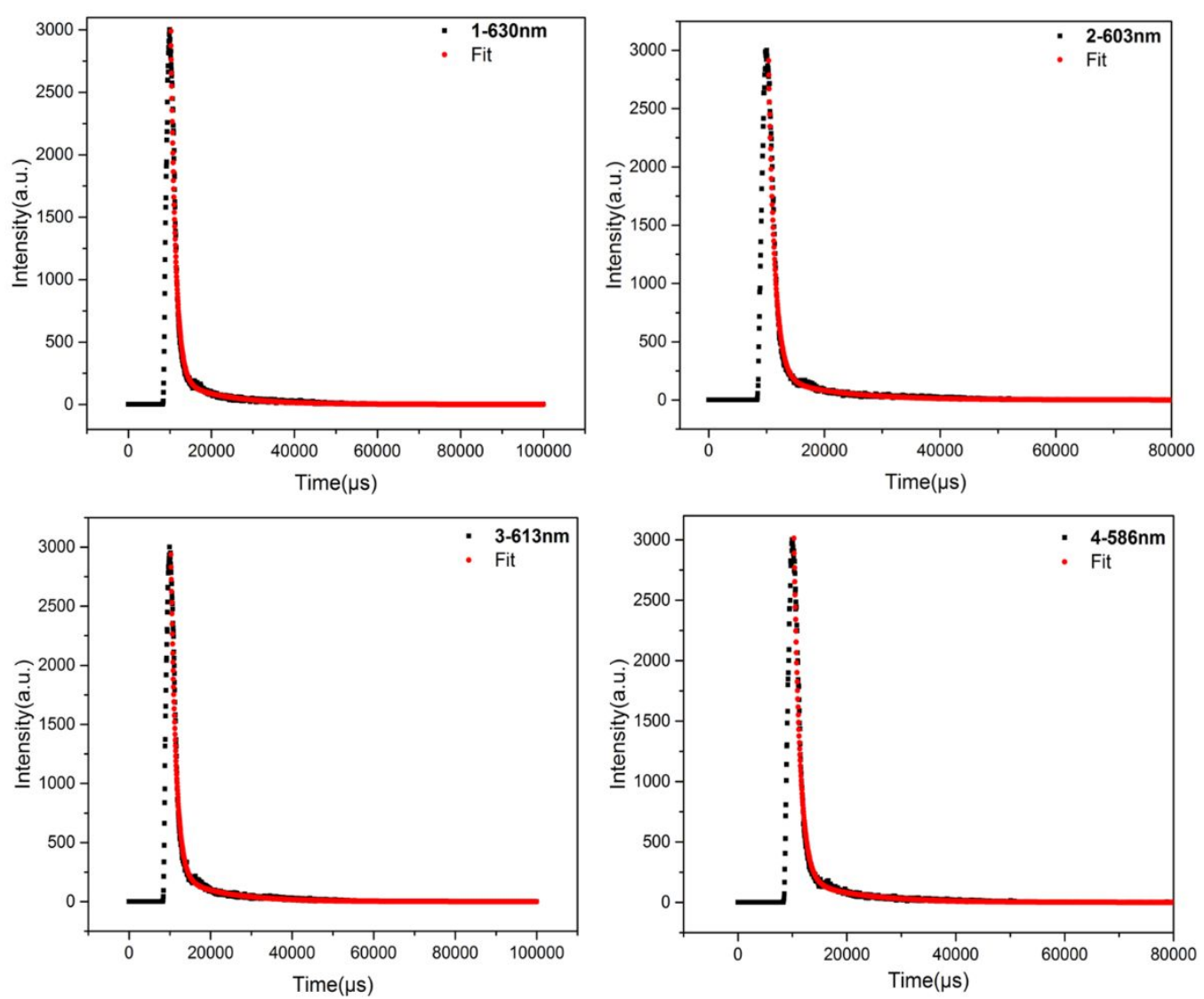

Fig. S6: Luminescence decays of the emissions at the long wavelengths for hybrids 1-4 by using microsecond lamp under ambient conditions 

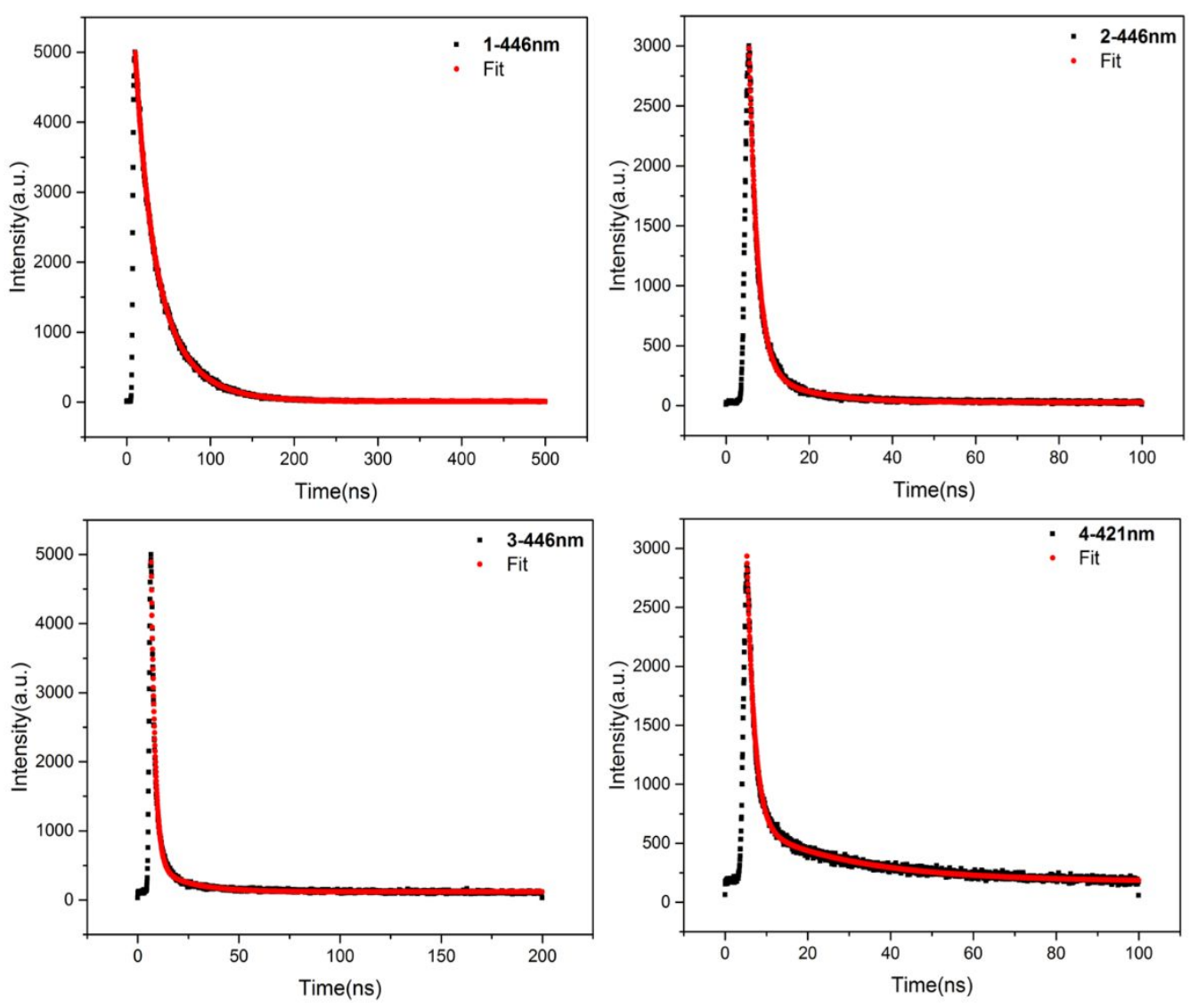

Fig. S7: Luminescence decays of the emissions at the short wavelengths for hybrids 1-4 by using nanosecond lamp under ambient conditions 